EXTENDED REPORT

\title{
Outcomes in persistent hyperplastic primary vitreous
}

\author{
A Hunt, N Rowe, A Lam, F Martin
}

Br J Ophthalmol 2005;89:859-863. doi: 10.1136/bjo.2004.053595

See end of article for authors' affiliations

......................

Correspondence to: Dr Adrian Robert Hunt, Sydney Eye Hospital, 8 Macquarie Street, Sydney, Australia 2000;

hunt_adrian@bigpond. com

Accepted for publication 5 October 2004

\begin{abstract}
Aims: To report outcomes in cases of persistent hyperplastic primary vitreous (PHPV) and examine factors predictive of visual acuity in patients selected for surgery.

Methods: Retrospective case series of 55 eyes in 50 patients presenting between 1990 and 2001 at the Children's Hospital, Westmead. 33 eyes underwent surgery aimed at visual rehabilitation.

Results: In surgical patients, median age at surgery was 77 days, and median age at final follow up was 28 months. Six (18\%) achieved a visual acuity at final follow up of $6 / 60$ or better and eight (24\%) achieved acuity of less than $6 / 60$ to counting finger vision inclusive. Those undergoing surgery before 77 days were approximately 13 times more likely to obtain a visual acuity of counting fingers or better than those operated later $(p=0.01)$. Neither posterior segment PHPV nor post-surgical glaucoma was a significant predictor of outcome in this selected group.

Conclusion: Early surgery aimed at rehabilitation markedly improves the likelihood of useful visual
\end{abstract} outcome in selected eyes with PHPV.
$\mathrm{P}$ ersistent hyperplastic primary vitreous (PHPV) causes considerable visual morbidity in affected eyes. Previous surgical series have documented variable visual and anatomical outcomes even with highly selected cases. ${ }^{1-13}$ Poor visual outcomes temper the decision to pursue surgery and ongoing visual rehabilitation.

We report on a series of 55 eyes diagnosed with PHPV managed surgically and conservatively over a 10 year period at the Children's Hospital at Westmead, Sydney. Data were analysed to determine factors predictive of visual outcome in eyes receiving surgical intervention aimed at visual rehabilitation.

\section{PATIENTS AND METHODS}

The medical records of patients with PHPV diagnosed by consultant paediatric ophthalmologists between January 1990 and March 2001 were reviewed retrospectively. The ethics committee of the Children's Hospital, Westmead, approved the research protocol. Departmental and hospital records were screened for potential PHPV, including cases coded as congenital cataract, microphthalmia, and congenital vitreous disorders. The inclusion criteria for this study involved documentation of typical anterior and/or posterior segment features of PHPV..$^{1-3}{ }^{12-15}$ These included microphthalmia, ${ }^{16}$ cataract, retrolental plaque, shallow anterior chamber, elongated ciliary processes, persistent hyaloid artery, retinal dysplasia, and retinal detachment. Diagnosis was made based on a combination of clinical, examination under general anaesthesia, and ultrasound findings. The difficult distinction between bilateral PHPV and Norrie disease was not defined by molecular genetic analysis. ${ }^{17}$ Preoperative ancillary tests including B-scan ultrasound with Doppler, computed tomography (CT), magnetic resonance imaging (MRI), and visual evoked potentials (VEP) were performed as directed by the treating ophthalmologist. Four surgeons performed or supervised all procedures.

In this study PHPV was categorised as anterior if a retrolental opacity, elongated ciliary processes, or cataract was present on clinical examination. PHPV was categorised as posterior when one or more of the following features was associated with an elevated vitreous membrane or stalk from the optic nerve: a retinal fold or retinal dysplasia, retinal detachment, or optic nerve hypoplasia.
Surgical interventions aimed at visual rehabilitation included lensectomy with or without anterior or total vitrectomy, and trabeculectomy. Cases with isolated anterior involvement or glaucoma were routinely selected for early surgery. Cases with minimal posterior involvement were selected upon the treating surgeon's discretion. Occlusion therapy combined with aphakic correction (contact lens, or spectacle if bilateral) followed surgery.

Visual acuity was assessed with age appropriate tests including Kays picture tests, Teller cards, Sheridan-Gardner, and Snellen tests. ${ }^{18}$ Age at surgery was calculated in days, and cases categorised into early and late surgery groups using the median value.

For patients receiving surgical intervention aimed at visual rehabilitation, final visual acuity was categorised into "useful" (counting finger vision or better) and "poor" (worse than counting fingers). Associations between clinical factors (age at surgery, sex, presence of posterior PHPV, and late glaucoma) and visual outcome were examined using $2 \times 2$ tables and logistic regression modelling. Statistical analyses were performed using SPSS, and significance defined as $\mathrm{p}<0.05$.

\section{RESULTS}

Fifty five eyes of 50 patients were included in the study, with 31 $(62 \%)$ males and $19(38 \%)$ females. Five $(10 \%)$ of the patients were diagnosed with bilateral PHPV and were assigned a single case number; 25 right eyes (45\%) and 30 left eyes (55\%) were included. Median age at diagnosis was 44 days. Three quarters were diagnosed within 90 days. PHPV was an isolated abnormality in most of the patients $(76 \%)$. Twelve patients had systemic abnormalities, seven of which were neurological and one case of Walker-Warburg syndrome (table 1). Fifteen eyes $(27 \%)$ had anterior segment involvement only. Thirty three $(60 \%)$ eyes had combined involvement. Seven (13\%) had purely posterior involvement, including hyaloid remnants (two eyes) with disc atrophy (one eye), large retrolental/central vitreous masses (five eyes) with vitreous haemorrhage (one eye), and retinal detachment (three eyes). Five $(71 \%)$ of the

Abbreviations: $\mathrm{CT}$, computed tomography; MRI, magnetic resonance imaging; PFV, persistent fetal vasculature; PHPV, persistent hyperplastic primary vitreous; VEP, visual evoked potentials 


\begin{tabular}{ll} 
Table 1 & Additional diagnoses in 12 patients \\
\hline Case No & Condition \\
\hline 18 & Bilateral deafness, behavioural abnormalities \\
21 & Ipsilateral retinopathy of prematurity \\
23 & Ipsilateral facial asymmetry \\
24 & Tracheomalacia, polymicrogyria with seizures \\
28 & Walker-Warburg syndrome, hydrocephaly, polycythaemia \\
30 & Patent ductus arteriosus \\
32 & Microcephaly \\
38 & Developmental delay, syndactyly, ear/nose abnormalities \\
41 & Microcephaly \\
45 & Ipsilateral hemifacial dysmorphism \\
48 & Ipsilateral hypoplasia of right optic nerve, chiasm, carotid \\
50 & artery and cerebrum \\
\hline
\end{tabular}

eyes with pure posterior PHPV had neurological abnormalities listed in table 1.

\section{Early surgical intervention aimed at visual rehabilitation}

Thirty three $(60 \%)$ eyes underwent early surgery directed at visual rehabilitation (table 2). Median age at diagnosis was 40 days. Median age at surgery was 77 days, and one quarter underwent surgery in the first month of life. Median age at final follow up was 28 months.

Signs of incipient or actual angle closure glaucoma complicated the clinical course in seven $(23 \%)$ eyes of this group. Two eyes in one patient underwent initial trabeculectomy because of congenital glaucoma with cloudy corneas. Subsequent visualisation of the extent of bilateral disease in this patient combined with significant co-morbidities led the clinician to pursue a more conservative approach. Five eyes were complicated by glaucoma after lensectomy. Late trabeculectomy was performed on two eyes (final VA: 1/24, NLP), medical management undertaken in two eyes $(1 / 60$, NLP), and photocycloablation performed in one eye (NLP). Strabismus was documented in $22(67 \%)$ patients and six of these underwent eye muscle surgery. Retinal detachment was documented in three (9\%) eyes and phthisis documented in another four $(12 \%)$ eyes in this group.

Subsequent interventions in this group included pupilloplasty in two eyes, YAG laser capsulotomy (one eye), surgical capsulotomy (one eye), and secondary intraocular lens implantation (two eyes). Two eyes were enucleated and three eyes had socket moulding for fitting of prostheses.

Visual acuity data were available for 31 eyes (94\%) in this group at final follow up. Six ( $18 \%)$ achieved a visual acuity at final follow up of 6/60 or better. Eight (24\%) achieved acuity of less than $6 / 60$ to counting finger vision inclusive. Seven $(21 \%)$ had hand movement to light perception vision inclusive, and 11 (33\%) had no light perception or had undergone enucleation.

Cases 21, 24, and 28 were excluded from statistical analysis, as visual outcome may have been affected by ocular or neurological conditions in addition to PHPV. There was no significant correlation between "useful" vision (defined as counting fingers at 1 metre or better acuity) at final follow up, and sex, eye, location of PHPV, or post-surgical glaucoma in regression analyses. After adjustment for post-surgical glaucoma and posterior segment PHPV, surgery before 77 days of age was found to strongly predict "useful" vision (OR 13.0, $\mathrm{p}=0.01$ ).

\section{Conservative management}

Twenty two eyes were managed without early surgical intervention aimed at visual rehabilitation (table 3 ).
Median age at diagnosis was 55 days. Median age at final follow up was 13 months.

Retinal detachment was documented in six $(28 \%)$ eyes in this group. Angle closure glaucoma was documented in one eye. Strabismus was documented in six $(28 \%)$ patients in this group. Strabismus surgery was performed in one patient in this group. Moulding for prosthesis fitting was performed on three eyes. Probing was performed for nasolacrimal duct obstruction for one patient.

Final visual acuity was available for 20 of these patients. One $(4 \%)$ achieved a visual acuity at final follow up of $6 / 48$ or better. One $(4 \%)$ achieved acuity of $6 / 60$ to counting finger vision inclusive. Six $(28 \%)$ had hand movement to light perception vision inclusive. Ten $(45 \%)$ had no light perception and one had undergone enucleation as a primary procedure.

\section{DISCUSSION}

Persistent hyperplastic primary vitreous was first described by Reese in 1955 in his Jackson Memorial Lecture. ${ }^{14}$ Representing a form of persistent fetal vasculature (PFV), Goldberg has suggested the terminology change to better reflect the pathology. ${ }^{18}$ The exact aetiology remains unknown. Characteristically PHPV presents as unilateral leucocoria with variably severe microcornea or microphthalmia. ${ }^{13}$ PHPV is usually found in otherwise healthy full term infants, however, it has been described in association with systemic and, in particular, neurological abnormalities. ${ }^{19}$ Severity can range from pupillary strands or an isolated Mittendorf dot, to more severe forms with retrolenticular membranes, retinal dysplasia, or detachment. ${ }^{1315182021}$

PHPV management is based on the extent of anterior and posterior segment involvement. Posterior PHPV has been associated with a poor visual outcome in previous studies. ${ }^{1-13}$ Including all patients in our study, the absence of posterior involvement predicted counting finger or better vision but this failed to reach statistical significance (OR 1.6, $\mathrm{p}=0.44$ ). For patients receiving visual rehabilitation surgery, presence of posterior segment involvement was found not to be associated with visual acuity at final follow up $(p=0.46)$. This failure to achieve statistical significance may be due to small patient numbers, short follow up time, and/or a selection for surgery of patients with only minimal posterior segment disease.

Pollard stated that age at presentation is a major predictor of visual outcome. ${ }^{3}$ Karr and Scott found surgical patients achieving 20/200 or better acuity presented at a mean age of 2.4 months, whereas those that achieved 20/300 or worse presented at a mean age of 4.3 months. ${ }^{5}$ Our study showed surgery before 77 days was associated with useful vision (OR $13, \mathrm{p}=0.01)$. This provides a useful prognostic factor in case selection that concurs with previous anecdotal reports. Comparing results with Karr and Scott's series, eyes achieving $6 / 60$ or better in our study had mean age at surgery of 41 days ( 1.3 months), excluding one patient operated at age 15 months who achieved 6/48. Mean age at surgery for patients achieving worse than 6/60 was 121 days (4.0 months).

Other factors examined in the statistical analysis (sex, laterality, and late glaucoma) were found not to be significantly associated with visual outcomes, although a possible trend for poor final acuity in males was found after multivariate adjustment (odds ratio 0.08, $\mathrm{p}=0.09$ )). However, the limited size of our series means all calculated associations are unstable to small changes in frequencies in any category of predictor or outcome factor. These low frequencies are also reflected in the large confidence intervals obtained for significant associations. The authors also recognise other factors such as compliance with occlusion 
Table 2 Surgical rehabilitation patient data

\begin{tabular}{|c|c|c|c|c|c|c|c|c|c|c|c|}
\hline $\begin{array}{l}\text { Case } \\
\text { No }\end{array}$ & Sex & Eye & $\begin{array}{l}\text { Age at } \\
\text { diagnosis } \\
\text { (months) }\end{array}$ & $\begin{array}{l}\text { Best preoperative } \\
\text { visual acuity }\end{array}$ & $\begin{array}{l}\text { PHPV } \\
\text { type }\end{array}$ & $\begin{array}{l}\text { Indications } \\
\text { for surgery }\end{array}$ & Surgery & $\begin{array}{l}\text { Age at } \\
\text { surgery } \\
\text { (months) }\end{array}$ & $\begin{array}{l}\text { Sequelae and } \\
\text { clinical course }\end{array}$ & $\begin{array}{l}\text { Age at final } \\
\text { follow up } \\
\text { (months) }\end{array}$ & $\begin{array}{l}\text { Visual acuity at } \\
\text { final follow up }\end{array}$ \\
\hline 1 & $M$ & RE & 2 & Vague LP & $A$ & C RP & $\mathrm{L} / \mathrm{V}$ & 4 & & 30 & $F \& F$ \\
\hline 2 & $\mathrm{~F}$ & $\mathrm{LE}$ & 2 & Vague LP & $A / P$ & C RP & L/V & 3 & $\begin{array}{l}\text { Indrawing of ciliary } \\
\text { processes, LXT small }\end{array}$ & 14 & Vague LP \\
\hline 3 & $M$ & RE & 3 & Poorly F\&F & $\mathrm{A} / \mathrm{P}$ & C & L & 9 & $\begin{array}{l}\text { Post-PHPV noted } \\
\text { postoperatively, RET }\end{array}$ & 8 & Poorly fixes \\
\hline 4 & $M$ & RE & 1 & Vague LP & $A / P$ & $C G$ & L & 13 & Glaucoma, trab, RD & 16 & NLP \\
\hline 5 & $M$ & $\mathrm{LE}$ & 0 & NA & A & C RP & L/V & 0 & & 42 & $3 / 18$ Kays \\
\hline 6 & $\mathrm{~F}$ & $\mathrm{RE}$ & 0 & Poorly F\&F & A & C RP & $\mathrm{L} / \mathrm{V}$ & 1 & $\begin{array}{l}\text { RXT variable, } \mathrm{CL} \\
\text { compliance poor }\end{array}$ & 43 & $1 / 60$ SGSL \\
\hline 7 & $\mathrm{~F}$ & RE & 0 & NA & $A / P$ & C RP & $\mathrm{L} / \mathrm{V}$ & 1 & $\begin{array}{l}\text { Pupil closure, } \\
\text { pupilloplasty, RET }\end{array}$ & 12 & 6/570Teller \\
\hline 8 & M & LE & 1 & NA & $A / P$ & C RP & L/V & 1 & $\begin{array}{l}\mathrm{RD}, \mathrm{CL} \text { compliance } \\
\text { poor }\end{array}$ & 116 & $\mathrm{LP}$ \\
\hline 9 & $M$ & RE & 14 & Poorly F\&F & $A / P$ & C RP & $\mathrm{L} / \mathrm{V}$ & 15 & $\begin{array}{l}\mathrm{CL} \text { compliance poor, } \\
\text { secondary IOL }\end{array}$ & 25 & 6/48 Teller \\
\hline 10 & $\mathrm{~F}$ & LE & 2 & NA & $A / P$ & C & L & 2 & RET & 107 & 6/48 Snellen \\
\hline 11 & $\mathrm{~F}$ & RE & 10 & Poorly F\&F & $A / P$ & C RP & L/V & 10 & $\begin{array}{l}\text { Pupil closure, } \\
\text { pupilloplasty, RET }\end{array}$ & 42 & $\mathrm{LP}$ \\
\hline 12 & $M$ & LE & 2 & No F\&F & $A / P$ & C RP & $\mathrm{L} / \mathrm{V}$ & 2 & Phthisis & 23 & Prosthesis \\
\hline 13 & $M$ & LE & 4 & No F\&F & $A / P$ & C RP AC & $\mathrm{L} / \mathrm{V}$ & 4 & Glaucoma, phthisis & 22 & NLP \\
\hline 14 & $M$ & LE & 1 & Poorly F\&F & $A / P$ & C & $\mathrm{L}$ & 2 & LET small & 32 & 6/36 Teller \\
\hline 15 & $M$ & $\mathrm{RE}$ & 0 & Poorly F\&F & A & C RP & L/V & 2 & RXT & 1 & $\mathrm{~F} \& \mathrm{~F}$ \\
\hline 16 & $\mathrm{~F}$ & $\mathrm{LE}$ & 4 & 6/480 Teller & $A / P$ & C RP & L/V & 5 & LET, squint surgery & 95 & $3 / 36$ Snellen \\
\hline 17 & $\mathrm{~F}$ & $\mathrm{LE}$ & 2 & NA & A & C & $\overrightarrow{\mathrm{L}}$ & 2 & $\begin{array}{l}\mathrm{LXT}, \mathrm{CL} \text { compliance } \\
\text { poor, secondary IOL }\end{array}$ & 54 & $3 / 36$ Kays \\
\hline 18 & $M$ & LE & 0 & Poorly F\&F & $A$ & C RP & $\mathrm{L} / \mathrm{V}$ & 4 & LXT, enucleation & 20 & NLP \\
\hline 19 & M & RE & 1 & NA & $A$ & C RP & $\mathrm{L} / \mathrm{V}$ & 1 & $\begin{array}{l}\text { YAG laser } \\
\text { capsulotomy }\end{array}$ & 22 & 6/36 Teller \\
\hline 20 & $\mathrm{~F}$ & LE & 7 & Poorly F\&F & $A / P$ & C & L & 6 & $\begin{array}{l}\text { LET, CL compliance } \\
\text { poor }\end{array}$ & 123 & LP \\
\hline 21 & M & $\mathrm{LE}$ & 2 & NA & $A / P$ & C & L & 6 & $\begin{array}{l}\text { LET, squint surgery, } \\
\text { threshold ROP, } \\
\text { cryotherapy, } \\
\text { glaucoma }\end{array}$ & 17 & 1.5/60 Teller \\
\hline 22 & $\mathrm{~F}$ & LE & 4 & NA & $A / P$ & C RP & $\mathrm{L} / \mathrm{V}$ & 4 & LET variable & 1 & NA \\
\hline 23 & M & RE & 1 & NA & $A / P$ & C RP AC & L/V & 1 & $\begin{array}{l}\text { Glaucoma, } \\
\text { photocycloablation }\end{array}$ & 28 & NLP \\
\hline 24 & $M$ & RE & 0 & NA & A & C & L & 1 & $\begin{array}{l}\text { RET, squint surgery, } \\
\text { capsulotomy }\end{array}$ & 13 & NLP \\
\hline 25 & M & LE & 0 & 6/240 Teller & $A / P$ & C & L & 10 & RET, RD & 44 & NLP \\
\hline 26 & $\mathrm{~F}$ & RE & 3 & NA & A & C RP & $\mathrm{L} / \mathrm{V}$ & 5 & $\begin{array}{l}\mathrm{RHT} / \mathrm{XT} \text {, squint } \\
\text { surgery }\end{array}$ & 104 & NA \\
\hline 27 & $M$ & LE & 0 & NA & $A / P$ & C & L & 1 & $\begin{array}{l}\text { Dead (PUO) at age } \\
14 \text { months }\end{array}$ & 8 & $6 / 120$ Teller \\
\hline $28 R$ & $\mathrm{~F}$ & RE & 0 & NA & $A / P$ & G & Trab. & NA & $\begin{array}{l}\text { Presented with } \\
\text { glaucoma }\end{array}$ & 8 & NLP \\
\hline $28 \mathrm{~L}$ & $\mathrm{~F}$ & LE & 0 & NA & $A / P$ & G & Trab. & NA & $\begin{array}{l}\text { Presented with } \\
\text { glaucoma }\end{array}$ & 8 & NLP \\
\hline $29 L$ & $\mathrm{~F}$ & $\mathrm{LE}$ & 1 & Poorly F\&F & $A / P$ & C RP & $\mathrm{L} / \mathrm{V}$ & 1 & $\mathrm{AET}$, squint surgery & 52 & $1 / 18$ SGSL \\
\hline $29 R$ & $\mathrm{~F}$ & RE & 1 & Poorly F\&F & $A / P$ & C RP & $\mathrm{L} / \mathrm{V}$ & 1 & $\begin{array}{l}\text { AET, squint surgery, } \\
\text { glaucoma, trab age } \\
4\end{array}$ & 52 & 1/24 SGSL \\
\hline $30 R$ & $\mathrm{~F}$ & RE & 2 & NA & $A / P$ & C RP & $\mathrm{L} / \mathrm{V}$ & 4 & $\begin{array}{l}\mathrm{RD} \text {, phthisis, } \\
\text { enucleation }\end{array}$ & 80 & NLP \\
\hline $31 \mathrm{~L}$ & $\mathrm{~F}$ & LE & 1 & LP & A & C & L & 2 & & 63 & $3 / 24$ SGSL \\
\hline
\end{tabular}

LP, light perception; NLP, no light perception; F\&F, fixing and following; $A$, anterior PHPV; $P$, posterior; $C$, cataract; $R P$, retrolental plaque; $G$, glaucoma; $A C$, shallow anterior chamber; Trab, trabeculectomy; L, lensectomy; V, vitrectomy; RET, right esotropia; LET, left esotropia; RXT, right exotropia; RHT, right hypertropia; AET, alternating esotropia; RD, retinal detachment; ROP, retinopathy of prematurity; CL, contact lens; secondary IOL, secondary intraocular lens; SGSL, SheridanGardner single letters.

and correction of refractive errors may influence outcome, but precise measurement of all potential confounders was limited by the retrospective nature of the study. Caution must be exercised in interpreting results given treatment groups (early versus late surgery) were not randomly allocated.

The difficult task of managing anisometropic amblyopia in neonatal aphakia is cited as a major factor contributing to poor visual outcome. ${ }^{22}$ Anteby et al concluded that intraocular lens implantation may be a favourable and beneficial option for the management of children with unilateral PHPV in their series of 89 children. ${ }^{23}$ Thirty of the 61 eyes in that study underwent cataract extraction/vitrectomy with intraocular lens implantation. High intraocular pressure and glaucomatous changes were observed less commonly with pseudophakia ( $8 \%$ ) compared to aphakia $(23 \%)$, and the incidence of NLP was $10 \%$ and $43 \%$ respectively. Our predominantly aphakic experience has been similar with a $23 \%$ incidence of glaucoma, and final VA of NLP in $32 \%$. The two secondary pseudophakic cases in our series were not complicated by glaucoma and achieved 3/36 Kays and 6/48 Teller VA.

Surgical intervention has previously been recommended for globe preservation in PHPV complicated by progressive retinal detachment, angle closure glaucoma, and recurrent intraocular haemorrhage..$^{1-4} 14{ }^{15}{ }^{24}$ In the surgical group eight eyes were complicated by glaucoma or retinal detachment, and seven of these had no light perception at final follow up. Four eyes underwent trabeculectomy with three having no 
Table 3 Conservative management patient data

\begin{tabular}{|c|c|c|c|c|c|c|c|c|c|}
\hline Case & Sex & Eye & $\begin{array}{l}\text { Age at } \\
\text { diagnosis } \\
\text { (months) }\end{array}$ & $\begin{array}{l}\text { Visual acuity at } \\
\text { presentation }\end{array}$ & $\begin{array}{l}\text { PHPV } \\
\text { type }\end{array}$ & $\begin{array}{l}\text { Contraindications } \\
\text { to early surgery }\end{array}$ & Miscellaneous & $\begin{array}{l}\text { Age final } \\
\text { follow up } \\
\text { (months) }\end{array}$ & $\begin{array}{l}\text { Visual acuity at } \\
\text { final follow up }\end{array}$ \\
\hline $30 \mathrm{~L}$ & $\mathrm{~F}$ & LE & 2 & NLP & $\mathrm{A} / \mathrm{P}$ & SA SP & $\begin{array}{l}\text { Corneal diameter } 7 \mathrm{~mm} \text {, patent } \\
\text { hyaloid artery }\end{array}$ & 80 & NLP \\
\hline $31 R$ & $\mathrm{~F}$ & RE & 1 & LP & A & G SA SP & $\begin{array}{l}\text { Glaucoma at presentation, } \\
\text { phthisis, prosthesis }\end{array}$ & 63 & NLP \\
\hline $32 \mathrm{~L}$ & $M$ & LE & 2 & $\mathrm{LP}$ & $P$ & SP & $\begin{array}{l}\text { Large retrolental mass, } \\
\text { rsmicrocephaly }\end{array}$ & 2 & $\mathrm{LP}$ \\
\hline $32 \mathrm{R}$ & M & RE & 2 & Vague LP & $P$ & $\mathrm{SP}$ & $R D$, microcephaly & 2 & Vague LP \\
\hline 33 & $\mathrm{~F}$ & $\mathrm{LE}$ & 0 & NLP & $A / P$ & SA SP & Microphthalmos, prosthesis & 95 & NLP \\
\hline 34 & $\mathrm{~F}$ & RE & 2 & Vague LP & $A / P$ & SP & Patent hyaloid artery to disc & 2 & Vague LP \\
\hline 35 & M & RE & 0 & NLP & $A / P$ & SA SP & $\begin{array}{l}\text { Shallow anterior chamber, AP } \\
14 \mathrm{~mm}\end{array}$ & 1 & NLP \\
\hline 36 & $M$ & RE & 0 & NLP & A & SA & $\begin{array}{l}\text { Shallow anterior chamber, late } \\
\text { lensectomy for glaucoma }\end{array}$ & 17 & NA \\
\hline 37 & $\mathrm{~F}$ & $\mathrm{LE}$ & 5 & Poorly F\&F & $A / P$ & $\mathrm{SP}$ & $\begin{array}{l}\text { Patent hyaloid artery to disc, } \\
\text { nystagmus }\end{array}$ & 47 & $3 / 60$ \\
\hline 38 & $\mathrm{~F}$ & RE & 0 & NA & $A / P$ & SA SP & $\begin{array}{l}\text { Ectopia lentis, retinal dysplasia, } \\
\text { L-ptosis and duct blockage } \\
\text { requiring probing. }\end{array}$ & 22 & NLP \\
\hline 39 & $M$ & RE & 0 & NA & $A / P$ & SA SP & $\begin{array}{l}\text { Corneal diameter } 8 \mathrm{~mm}, \mathrm{AP} \\
\mathrm{rs} 14 \mathrm{~mm}\end{array}$ & 11 & NLP \\
\hline 40 & $M$ & $\mathrm{LE}$ & 4 & NA & $A / P$ & SA SP & $\begin{array}{l}\text { Corneal diameter } 8 \mathrm{~mm}, \mathrm{AP} \\
\mathrm{rs} 13 \mathrm{~mm}\end{array}$ & 3 & NLP \\
\hline 41 & $M$ & LE & 1 & $\mathrm{LP}$ & $P$ & SP & $\begin{array}{l}\text { Hyaloid artery, hypoplastic disc, } \\
\text { roving nystagmus, microcephaly }\end{array}$ & 9 & $\mathrm{LP}$ \\
\hline 42 & $M$ & $\mathrm{LE}$ & 0 & NA & $P$ & SP & Progressive phthisis, enucleation & 37 & NLP \\
\hline 43 & M & $\mathrm{LE}$ & 3 & Good F\&F & $P$ & MP & $\begin{array}{l}\text { Hyaloid artery only, } \\
\text { developmental delay }\end{array}$ & 11 & $6 / 18$ \\
\hline 44 & M & LE & 3 & NA & $A$ & SA & $\begin{array}{l}\text { Shallow anterior chamber with } \\
\text { pupil bound down }\end{array}$ & 3 & NLP \\
\hline 45 & $\mathrm{~F}$ & RE & NA & NA & A & SA & Prosthesis & 13 & NLP \\
\hline 46 & $M$ & $\mathrm{LE}$ & 0 & Poorly F\&F & $A / P$ & SA SP & $\begin{array}{l}\text { Retrolental mass, AP } 12 \mathrm{~mm} \text { and } \\
\text { left ptosis, roving nystagmus }\end{array}$ & 56 & $\mathrm{LP}$ \\
\hline 47 & M & LE & 8 & NA & $A$ & SP & Retrolental mass & 8 & NA \\
\hline 48 & M & RE & 1 & Roving & $P$ & $\mathrm{SP}$ & Vitreous haemorrhage, RD & 14 & NLP \\
\hline 49 & $M$ & LE & 4 & NA & $P$ & $\mathrm{SP}$ & $\begin{array}{l}\text { Hyaloid artery and retrolental } \\
\text { mass, }\end{array}$ & 9 & NLP \\
\hline 50 & $M$ & RE & 2 & Poorly F\&F & $A / P$ & SA & $\begin{array}{l}\text { Shallow anterior chamber, } \\
\text { strabismus surgery }\end{array}$ & 59 & NLP \\
\hline
\end{tabular}

LP, light perception; NLP, no light perception; F\&F, fixing and following; $A$, anterior PHPV; P, posterior; MP, minimal posterior PHPV; SP, severe posterior PHPV not amenable to surgery; SA, severe anterior PHPV not amenable to surgery; G, glaucoma; RD, retinal detachment; AP, globe anteroposterior diameter; MRI, magnetic resonance imaging.

light perception at final follow up; none of these eyes went on to develop phthisis or required enucleation.

Mild PHPV can run a relatively benign natural course without surgery. ${ }^{25}$ Two patients in our series with an isolated hyaloid artery the only manifestation of PHPV achieved $\sim 6 / 18$ (Teller) at age 11 months and 3/60 (Sheridan-Gardner) at 4 years with conservative management. Surgery may be avoided if the visual axis is clear, anatomical anomalies are not progressive, and the anterior chamber angle is not compromised. ${ }^{126}$

Optimal timing of surgery aimed at visual rehabilitation is presumably governed by the early critical periods of visual development. ${ }^{27} 28$ The study findings support the value of early diagnosis and early intervention to maximise visual potential in selected patients.

\section{ACKNOWLEDGEMENTS}

We are grateful to the orthoptic staff at the Children's Hospital, Westmead, and also Professor Frank Billson, Dr Jeremy Smith, and Dr Michael Giblin, for their contribution to this article through patient care and management.

\section{Authors' affiliations}

A Hunt, N Rowe, F Martin, Department of Ophthalmology, The University of Sydney, New South Wales, Australia

A Lam, Department of Radiology, The Children's Hospital, Westmead, Sydney, Australia

\section{REFERENCES}

1 Goldberg MF. Persistent fetal vasculature (PFV): an integration of signs and symptoms associated with persistent hyperplastic primary vitreous (PHPV). LIV Edward Jackson Memorial Lecture. Am J Ophthalmol 1997;124:587-626.

2 Pollard Z. Results of treatment of persistent hyperplastic primary vitreous. Ophthalmic Surg 1991;22:48-52.

3 Pollard Z. Persistent hyperplastic primary vitreous: diagnosis, treatment and results. Trans Am Ophthalmol Soc 1997;95:487-549.

4 Smith RE, Maumenee AE. Persistent hyperplastic primary vitreous: results of surgery. Trans Am Acad Ophthalmol Otolaryngol 1974;78:911-25.

5 Karr DJ, Scott WE. Visual acuity results following treatment of persistent hyperplastic primary vitreous. Arch Ophthalmol 1986;104:662-7.

6 Acers TE, Coston TO. Persistent hyperplastic primary vitreous. Early surgical management. Am J Ophthalmol 1967;64:734-5.

7 Scott WE, Drummond GT, Keech RV, et al. Management and visual acuity results of monocular congenital cataracts and persistent hyperplastic primary vitreous. Aust N Z J Ophthalmol 1989;17:143-51

8 Federman JL, Shields JA, Altman B, et al. The surgical and non surgical management of persistent hyperplastic primary vitreous. Ophthalmology 1982;89:20-4

9 Alexandrakis G, Scott IU, Flynn HW, et al. Visual acuity outcomes with and without surgery in patients with persistent fetal vasculature. Ophthalmology 2000; 107: 1068-72.

10 Mittra RA, Huynh LT, Ruttum MS, et al. Visual outcomes following lensectomy and vitrectomy for combined anterior and posterior persistent hyperplastic primary vitreous. Arch Ophthalmol 1998;116:1190-4.

11 Reynolds JD. Visual acuity after treatment of persistent hyperplastic primary vitreous [letter]. Arch Ophthalmol 1986;104:1274-1277.

12 Dass $A B$, Trese MT. Persistent hyperplastic primary vitreous results. Ophthalmology 1999;106:280-4.

13 Hadda R, Font RL, Reeser F. Persistent hyperplastic primary vitreous. A clinicopathologic study of 62 cases and review of the literature. Surv Ophthalmol 1978;23:123-34.

14 Reese AB. Persistent hyperplastic primary vitreous. Jackson Memorial Lecture. Trans Am Acad Ophthalmol Otolaryngol 1955;59:271-86. 
15 Reese AB. Persistent hyperplastic primary vitreous. Am J Ophthalmol 1955:40:317-31.

16 Grignolo A, Riviera A. Biometry of the human eye from the sixth month of pregnancy to the tenth year of life. In: Vanysek J, ed. Diagnostica Ultrasonica in Ophthalmologia 1968:251-7.

17 Pendergast SD, Trese MT, Liu X, et al. Study of the Norrie disease gene in 2 patients with bilateral PHPV. Arch Ophthalmol 1998;116:381-2.

18 Taylor D, ed. History, examination and further investigation. In: Practical paediatric ophthalmology. 1st ed. Oxford: Blackwell Science, 1997:31-3.

19 Marshman WE, Jan JE, Lyons CJ. Neurologic abnormalities associated with persistent hyperplastic primary vitreous. Can J Ophthalmol 1999;34:17-22.

20 Duke-Elder SS, Cook C. The ocular vascular system. In: Duke-Elder SS, ed. System of ophthalmology, Vol III. Normal and abnormal development. Part I. Embryology. London: Henry Kimpton, 1963:179-209.
21 Pruett RC. The pleomorphism and complications of posterior primary vitreous. Am J Ophthalmol 1975;80:625-9.

22 Parks MM, Johnson DA, Reed GW. Long-term visual results and complications in children with aphakia. Ophthalmology 1993;100:826-41.

23 Anteby I, Cohen E, Karshai I, et al. Unilateral persistent hyperplastic primary vitreous: course and outcome. J AAPOS 2002;6:92-6.

24 Nankin SJ, Scott WE. Persistent hyperplastic primary vitreous. RotoExtraction and other surgical experience. Arch Ophthalmol 1977;95:240-3.

25 Maurer PA, Hiles DA. Unoperated persistent hyperplastic primary vitreous. Trans Am Acad Ophthalmol Otolaryngol 1987;38:518-22.

26 Gulati N, Eagle RC Jr, Tasman W. Unoperated eyes with persistent fetal vasculature. Trans Am Ophthalmol Soc 2003;101:59-64.

27 Raskind R. Persistent hyperplastic primary vitreous: necessity of early recognition and treatment. Am J Ophthalmol 1966;62:1072-6.

28 Von Noorden GK. Histological studies of the visual system in monkeys with experimental amblyopia. Invest Ophthalmol 1973;12:727-38.

Video reports

To view the video reports in full visit our website www.bjophthalmol.com and click on the link to the video reports.

- Zero Phaco Microincision Cataract Surgey: The Hacc Technique. K Vaitheeswaran, $S$ Gars, R Grover, M Nadar, S Sharma

- The presenting features of multiple sclerosis. VJM Barrett, J Walker, JS Elton

- Removal of INTACS: Stepped surgical complexity demonstrated with three cases. L Ilari, J C McAlister, D S Gartry

- The Nuclear Slide: A novel approach for unleashing the potential of the hydrodissection wave. A Naseri

- Giant pleomorphic adenoma of the lacrimal gland: pre- and post-operative function. A Jain, V I Nehru, U N Saikia, C E E Reddy

- Limbal-sparing lamellar keratoplasty. S L Watson, S Rauz, J Dart

- Bilateral Abducens Neuromyotonia. L H Ospina, N Aui-aree, D P Anderson

- Light to dark physiological variation in irido-trabecular angle width. G M Gazzard, P J Foster, D S Friedman, P T Khaw, S K L Seah

Video Suite: Triamcinolone-assisted vitrectomy

- Triamcinolone-assisted removal of the posterior hyaloid to repair retinal detachment due to macular hole in high myopia. A Ueno, H Enaida, Y Hata, T Nakamura, T Hisatomi, K Fujisawa, T Kubota, T Sakamoto, $T$ Ishibashi

- Triamcinolone acetonide-assisted Epiretinal Membrane Peeling. S D M Chen, C K Patel

- A suture technique to manage a case of severe early flap displacement after laser in situ keratomileusis. L Spadea, P Pantaleoni, G Bianco

- Reconstruction of the Ocular Surface in LOGIC Syndrome. E Moore, V Kumar, $J$ R Ainsworth, S Shah

- Laser Photocoagulation for Posterior Segment Intraocular Parasites. T Prabriputaloong, S Asawaphureekorn

- Feeder Vessel Treatment with High Speed ICG Angiography. D Stanescu-Segall, $G$ Coscas, F Coscas, G Soubrane

- Endoscopy to aid anterior segment surgery. J E Moore, A Sharm

- Penetrating ocular injury due to a fish hook: Surgical removal. SD M Chen,D Chiu, C K Patel

- Retinal Ganglion Cell Axon Response to Guidance Molecules. S F Oster and DW Sretavan

- Marin-Amat Syndrome. A Jogiya, C Sandy

- Excision of subcutaneous Dirofilariasis of the eyelid. D Mallick, TP Ittyerah

- Thixotropy: a novel explanation for the cause of lagophthalmos after peripheral facial nerve palsy. M Aramideh, J H T M Koelman, P P Devriese, F VanderWerf, JD Speelman

- Surgical revision of leaking filtering blebs with an autologous conjunctival graft. $K$ Taherian, A Azuara-Blanco

- Dipetalonema Reconditum in the human eye. T Huynh, J Thean, $R$ Maini 